\title{
Téoros
}

Revue de recherche en tourisme

\section{Le tout inclus}

\section{Ce tourisme qu'on dénigre individuellement mais qu'on choisit} " en masse »!

\section{Alain A. Grenier}

Volume 31, numéro 2, 2012

URI : https://id.erudit.org/iderudit/1020766ar

DOI : https://doi.org/10.7202/1020766ar

Aller au sommaire du numéro

\section{Éditeur(s)}

Université du Québec à Montréal

ISSN

0712-8657 (imprimé)

1923-2705 (numérique)

Découvrir la revue

Citer ce document

Grenier, A. A. (2012). Le tout inclus : ce tourisme qu'on dénigre

individuellement mais qu'on choisit " en masse "! Téoros, 31(2), 3-11.

https://doi.org/10.7202/1020766ar

Ce document est protégé par la loi sur le droit d'auteur. L’utilisation des services d'Érudit (y compris la reproduction) est assujettie à sa politique d'utilisation que vous pouvez consulter en ligne.

https://apropos.erudit.org/fr/usagers/politique-dutilisation/
Cet article est diffusé et préservé par Érudit.

Érudit est un consortium interuniversitaire sans but lucratif composé de l’Université de Montréal, l'Université Laval et l'Université du Québec à Montréal. Il a pour mission la promotion et la valorisation de la recherche. https://www.erudit.org/fr/ 


\title{
Le tout inclus \\ Ce tourisme qu'on dénigre individuellement mais qu'on choisit «en masse»!
}

\author{
Alain A. GRENIER, Ph.D. \\ Professeur au Département d'études urbaines et touristiques \\ École des Sciences de la gestion \\ Université du Québec à Montréal (ESG UQAM) \\ grenier.alain@uqam.ca
}

Le tout inclus est un sujet de débats passionnés : on aime ou on n'aime pas. Pourtant, ce tourisme qualifié de «Playa del anywhere» [Playa de n'importe où] (Selänniemi, 2001) (voir illustration 1), que l'on dénigre, individuellement ou en chœur, se vend pourtant... «en masse». Des destinations (les Caraïbes, la Méditerranée et plusieurs régions d'Asie) en vivent. À Hong Kong (7 millions d'habitants), pas moins de 1323 agences de voyages vivent principalement de la vente de produits de voyage de type tout inclus (Heung et $\mathrm{Chu}$, 2000 : 52; Lo et Lam, 2004 : 162). Les Asiatiques prisent en effet beaucoup le forfait de voyage qui permet notamment de contourner les barrières linguistiques à la destination. Cela permet ainsi de visiter des destinations lointaines (l'Europe et l'Amérique du Nord), dont la découverte des attraits constitue souvent un élément distinctif pour les Asiatiques. Les Européens ne sont pas en reste : ils pratiquent aussi le tout inclus mais vers les destinations «soleil». Les Européens choisissent principalement la Méditerranée et l'Asie. Les Danois, par exemple, effectuent plus du tiers de leurs séjours à l'étranger en tout inclus (Danmarks Statistik, 2009). Ils se joignent en majorité aux 10 millions de visiteurs qui fréquentent les îles Canaries, où le tourisme représente plus de $50 \%$ du produit intérieur brut (Scherrer et al., 2009). De l'autre côté de l'Atlantique, les Nord-Américains optent aussi pour le tout inclus en milieu balnéaire. Ainsi, entre 2010 et 2013, $44 \%$ des voyages effectués par des Canadiens et $58 \%$ de ceux réalisés par des Québécois à l'étranger se sont déroulés dans les Caraïbes (PMB, 2013).

C'est donc qu'il y en a passablement, parmi nous, qui choisissent le tout inclus et qui l'aiment. Aimer une forme de voyage ne veut pas nécessairement dire qu'elle soit bonne. Cependant, si elle répond aux besoins des masses, elle mérite de s'y attarder, d'autant que cette forme de voyage suscite critiques et controverses. Si l'on souhaite en corriger les aspects déficients, encore faut-il préalablement comprendre le phénomène et l'expérience qu'il propose. Or, malgré ses millions d'adeptes de par le monde, la recherche sur le tout inclus, comme phénomène de masse, est plutôt rare, notamment sur les considérations des voyageurs dans le choix du produit (Lauring, 2013 : 217) et en matière de gestion des sites. En fait, le tourisme est étudié soit dans son ensemble ou par ses (micro) phénomènes alternatifs, mais rarement en fonction de la prise en charge des vacanciers dans le cadre d'un forfait de vacances-voyage.

Le point de départ de notre réflexion - ce dossier spécial de Téoros - est sans contredit les reproches formulés de part et d'autre à l'endroit du tout inclus. On reproche à ce tourisme de masse de se laisser bercer dans l'abondance de son resort, de sa déshumanisation et de son indifférence à l'égard de l'hôte et de sa réalité (pauvreté, chômage, etc.). On lui attribue la détérioration des sites naturels, notamment en milieux côtiers, et la surenchère des titres immobiliers. On déplore les conditions de travail difficiles de la main-d'œuvre (longues heures, salaires minimum, bénéfices sociaux minimes, voire inexistants) et la fuite des capitaux des grandes chaînes hôtelières dont les sièges sociaux et les actionnaires se trouvent souvent bien loin des destinations et des communautés hôtes.

On reproche aussi à l'usager du tout inclus les abus et le gaspillage (voir illustration 2). Il est vrai que la formule engendre, chez certains vacanciers, une fausse impression d'abondance. Ainsi, le gavage alimentaire et à l'alcool et les familiarités sur le plan du civisme dans ses rapports avec les employés constituent quelques-uns des revers du tout inclus. Il n'est pas rare de voir plusieurs de ces touristes rois s'offusquer sur le vol qui les ramène à la maison lorsqu'ils réalisent qu'il n'y a plus de service de bar ouvert dans l'avion. Le semblant de supériorité économique et sociale qu'octroie l'expérience «tout inclus» expliquerait-il les abus de ces touristes? De ces questions et observations naît la nécessité d'une réflexion organisée.

L'objectif de ce dossier de Téoros n'est pas de défendre ou de condamner le tout inclus. Il est simplement de donner un espace pour permettre d'enrichir le débat et de trier entre les idées émiques (celles qui émergent d'une réflexion sans structure) et étiques (sans «h» - qui évoquent une 


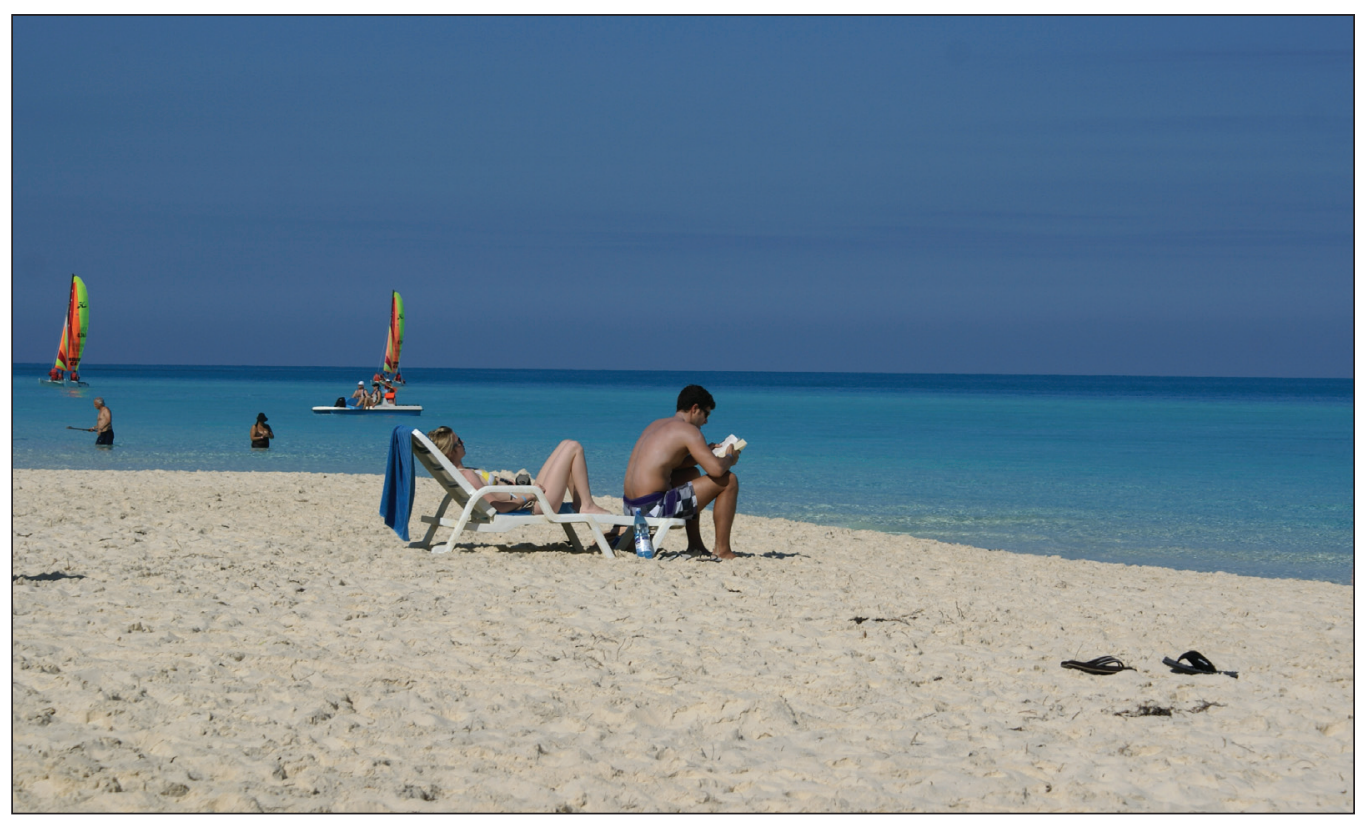

ILLUSTRATION 1 : Le tout inclus incarne le rêve d'un petit coin du paradis tropical à «Playa del anywhere» (photo : Alain A. Grenier). réflexion structurée). Le point de départ de notre réflexion est l'organisation du voyage et l'implication des touristes dans celle-ci puisque les voyageurs les moins impliqués ont tendance à être socialement perçus (à raison ou à tort) de façon plus péjorative que les autres. Tout est dans la compréhension de chacun.

\section{Un concept en évolution}

Comme tout phénomène socio-économique, le tout inclus n'est pas apparu tout à coup sous les traits qu'on lui connait. Il se situe dans le sillon du voyage organisé, alors que le touriste, dépourvu de l'expérience et des connaissances nécessaires au voyage, s'en remettait à l'expertise d'un voyagiste qui lui procurait toute la sécurité d'un séjour bien planifié. La différence se joue aujourd'hui davantage dans l'entassement des vacanciers dans des environnements fermés et déconnectés du milieu d'accueil.

Le tout inclus tire ses origines de centres de villégiature (des sortes de villages vacances) établis en Angleterre entre 1936 et 1966 par le Sud-Africain Billy Butlin, qui souhaitait offrir à ses compatriotes anglais moins fortunés l'occasion de vacances à bas prix où tous les services seraient «inclus». Concentrés au Royaume-Uni, ces centres de villégiature, appelés Butlins (aussi «Butlin's»), ont néanmoins traversé les mers pour s'établir aux Bahamas, en Espagne et même en France (dans une moindre mesure). La formule fut ensuite reprise et remaniée par le Belge Gérard Blitz avec le premier Club Méditerranée, établi à Mallorca, en Espagne, en 1950. Ici encore, l'objectif était d'offrir des vacances à peu de frais, mais en déplaçant le produit vers la destination soleil, à l'étranger.

L'évolution du tout inclus passe par différentes phases, elles-mêmes liées à des contextes très particuliers que les critiques ont tendance à oublier. Il prend d'abord racine dans les Caraïbes, plus spécifiquement en Jamaïque. Henry
(1989) identifie quatre phases marquantes de cette évolution. La première est associée aux décennies des années 1950 et 1960. Le développement touristique, alors perçu comme générateur d'emplois, est bien reçu dans les régions du sud où il devient un moyen de lutter contre la pauvreté. Les touristes, relativement peu nombreux comparativement à aujourd'hui, sont aussi plus fortunés et nécessitent des services de haute qualité (Henry, 1989 : 64). Les repas se prennent donc à l'hôtel, et le client paie à la carte. Les taxis, les artisans et les vendeurs de souvenirs constituent alors souvent les seules petites entreprises satellites à ce tourisme d'hôtel. Pour le divertissement, les vacanciers doivent s'en remettre aux boîtes de nuit, gérées aussi par des entrepreneurs locaux. Le succès de ces vacances tout inclus va bientôt se répandre comme une traînée de poudre. Avec l'émancipation des sociétés occidentales, à partir des années 1960, le phénomène du forfait de vacances marque plus que tout autre la croissance du tourisme de masse international (Shaw et Williams, 1994). On aime son prix et la sécurité qu'il promet (Lauring, $2013: 217$ ).

La seconde phase, pendant les années 1970, engendre la massification du tourisme balnéaire - et la seconde vague de son développement (Henry, 1989). La compétition entre les diverses lignes aériennes entraîne une réduction des coûts de transport (Lo et Lam, $2004: 162$ ). Avec plus de voyageurs, le prix du produit balnéaire devient encore plus accessible, attirant ainsi des touristes moins fortunés. Ces derniers délaissent les services de l'hôtel, trop coûteux, optant plutôt pour les restaurants bon marché des résidents (Henry, 1989 : 64). Cette tendance encourage les communautés à ouvrir plus de restaurants, souvent de type restauration rapide (pizzerias et casse-croûte, par exemple). De même, l'augmentation du nombre de vendeurs de souvenirs en Jamaïque, durant ces mêmes années, serait attribuable à un contexte économique difficile où chacun tentait, par toutes sortes de moyens, de 
ILLUSTRATION 2 : La formule de buffet, très populaire dans les tout inclus, facilite la logistique des repas mais crée, du même souffle, une impression d'abondance dont il est facile d'abuser (photo : Alain A. Grenier).

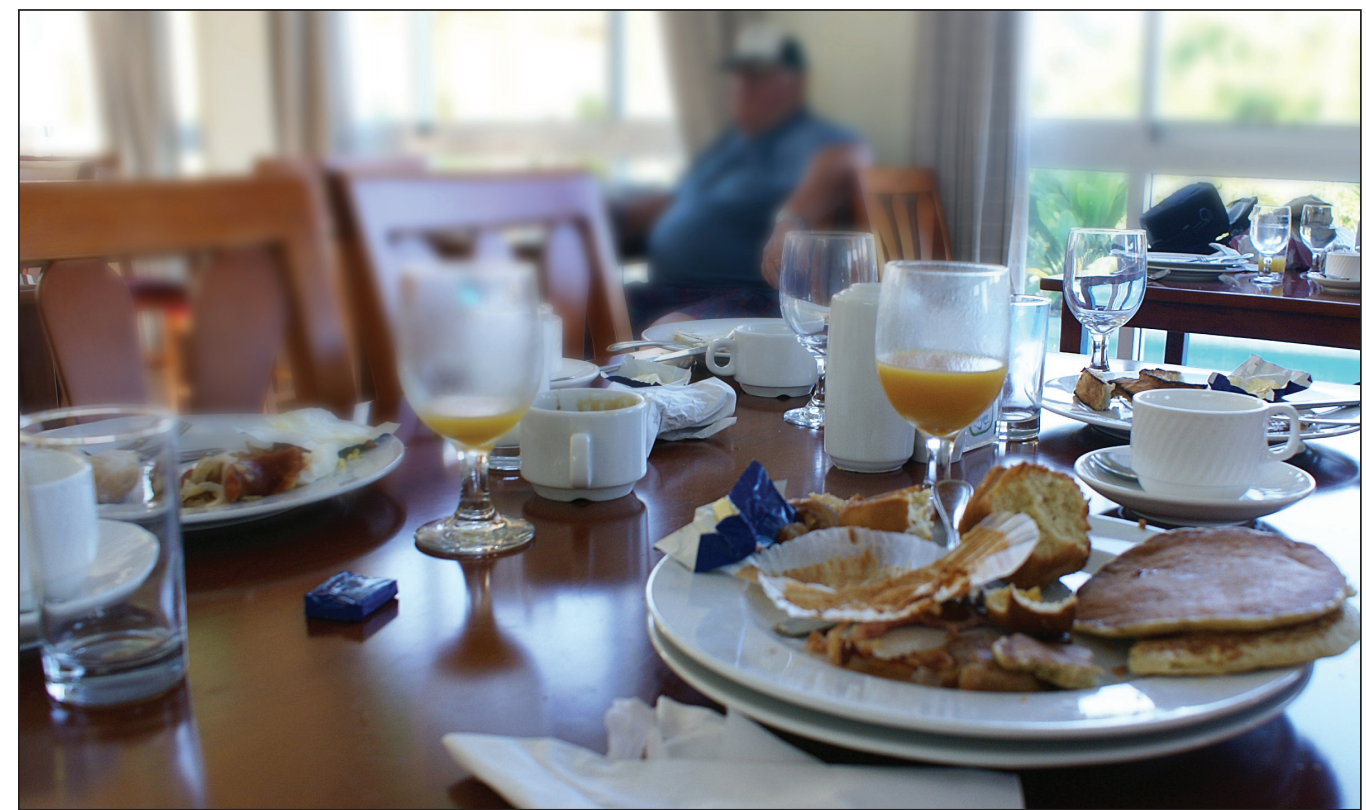

solliciter l'argent neuf directement auprès des (touristes) étrangers (Henry, 1989 : 64). Cet engouement local, notamment pour la pour la restauration, se traduit par la croissance importante du nombre de ces petites entreprises durant les années 1980.

Rien ne laisse pourtant présager le nuage qui plane à l'horizon : le tout inclus devient parallèlement, durant cette troisième étape, le modèle de vacances soleil prédominant. En effet, ces touristes étrangers, s'ils sont encore plus nombreux, sont aussi moins nantis et commencent à regarder de plus près les coûts associés à leurs vacances. Flairant ce changement de courant, certains hôteliers commencent à opter, à la fin des années 1970, pour une formule inclusive, jouant la carte du compromis entre la variété et la qualité. La formule, économiquement accessible à un plus large public, gagnera rapidement en popularité. En revanche, elle enclavera les touristes dont les sorties du complexe hôtelier diminuent inévitablement, notamment pour les besoins de restauration dorénavant comblés à meilleur prix à l'hôtel.

Situés en bordure de la mer, souvent au prix de la délocalisation des populations locales, les hôtels de type tout inclus concentrent tous les services de villégiature sur un site donné, et fermé (dans certains pays d'Amérique latine, sous protection armée).

Une dernière et quatrième étape de l'histoire du tout inclus s'écrit depuis les années 1990 : celle des impacts, souvent négatifs, générés par ces enclaves à touristes sur les milieux sociaux (Henry, 1989). En réponse à ces critiques, certains tout inclus réagissent pour altérer la formule. Par exemple, certains se sont associés à des restaurateurs locaux pour offrir aux touristes l'occasion de repas hors des murs de l'hôtel, par le biais de cartes prépayées en faveur de restaurants participants. Toutefois, les problèmes du tout inclus vont bien au-delà de la logistique des repas. Le problème de gestion relève aussi du concept même de l'expérience offerte.

\section{Le concept de tout inclus : variations sur le thème du séjour organisé}

Le tout inclus est l'un des produits offerts dans la famille des forfaits des voyages organisés. Un forfait comprend une combinaison d'au moins deux des éléments suivants : le transport, l'hébergement, la restauration, l'accès aux attraits à la destination, les assurances et d'autres services (Middleton, 1991 : 185; Lo et Lam, 2004 : 172). Il en résulte un produit standardisé pour les masses et dont l'offre peut-être continuellement répétée (Lo et Lam, 2004 : 162). En ce sens, il se définit en opposition au voyage à la carte ou au voyage totalement autonome (en anglais fully independent travel - FIT, Yamamoto et Gill, 1999: 134), où le vacanciers voit lui-même à assembler les différentes composantes de son voyage, achetées séparément.

Wong et Kwong (2004 : 581) identifient deux types de forfaits : de base et tout inclus (en anglais : all-inclusive tour). Le premier combine en un produit le transport et l'hébergement tandis que le second, comme son nom l'indique, comprend «tout». Ils se distinguent non seulement par leurs composantes, mais selon le degré de liberté et de flexibilité des touristes dans la prise d'arrangements qui les lie à la destination.

De plus, le tout inclus prend différente formes. Il y a ce voyage "organisé» (charter tourism ou group package tour) aussi appelé voyage guidé (guided tour). Ce type de voyage, mobile, prend la forme des séjours guidés en autocar qui assemblent dans un même forfait le transport, l'hébergement, la restauration et les visites guidées, appliquées à une ou plusieurs régions d'une destination (l'Europe en 14 jours, par exemple). Ce forfait s'adresse davantage à des groupes formés avant le départ. Il est généralement considéré par les touristes comme la méthode la plus rationnelle et efficace pour découvrir un maximum de lieux à l'intérieur d'un seul séjour d'une durée déterminée (Enoch, 1996). Puis il y a le tout inclus «fixe» — un produit de voyage clé 


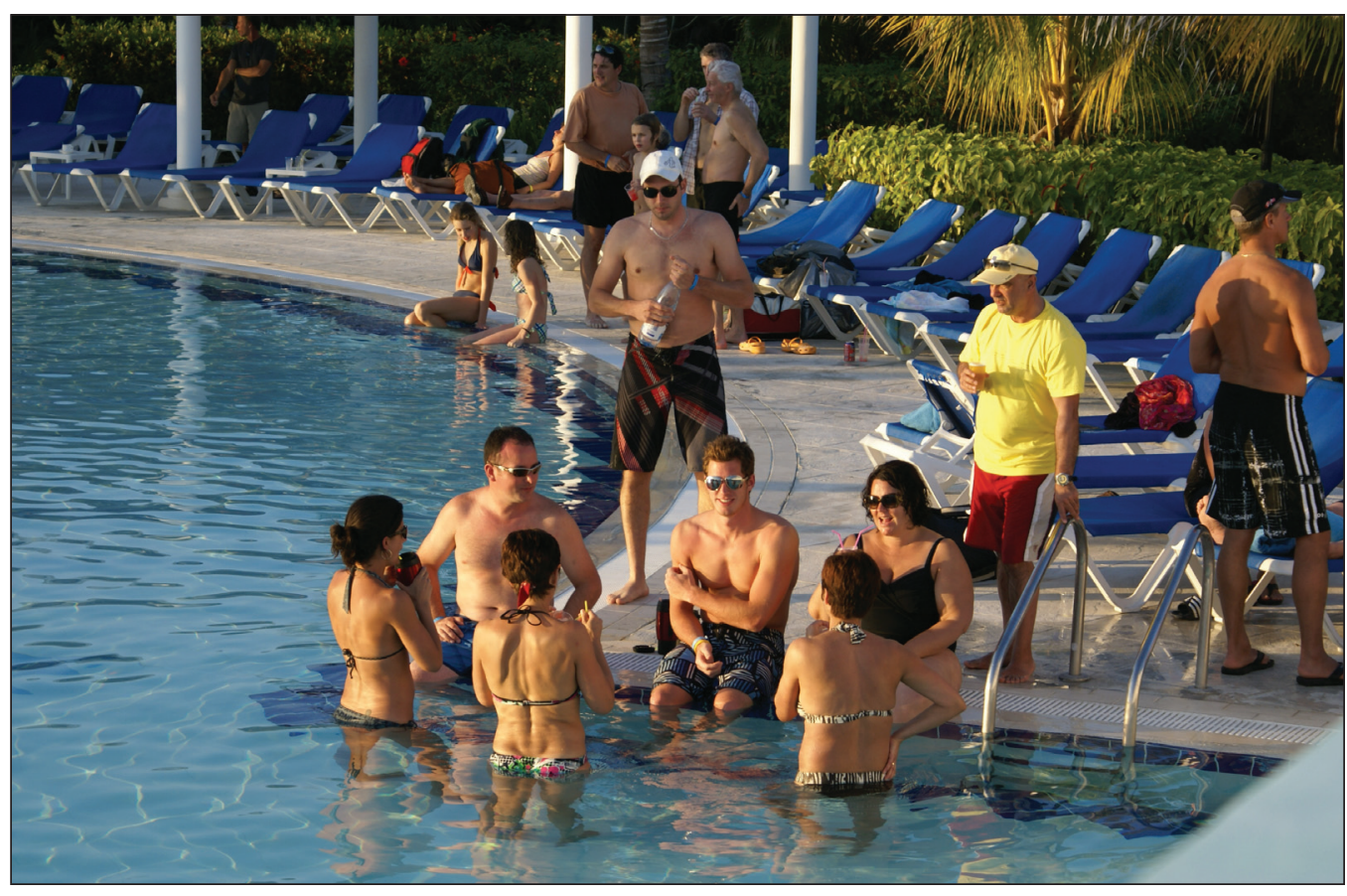

ILLUSTRATION 3 : Le contexte du milieu de repos, relativement fermé, procure aux membres d'une même culture un sentiment de sécurité en milieu étranger (photo : Alain A. Grenier). en main où tous les préparatifs et la logistique de l'organisation du voyage (transport), du séjour (en hébergement fixe) et du déroulement du voyage (alimentation, divertissements et une large part des activités récréatives) sont pris en main par le voyagiste et payé par le client préalablement au départ. Ainsi, tandis que le forfait devient une forme de voyage à la carte, le tout inclus propose une formule complètement inclusive dans laquelle l'usager est entièrement pris en charge par le voyagiste.

Principalement associé aux destinations soleil et aux croisières, le tout inclus fait aussi partie de l'offre des pays moins tempérés qui proposent également aux visiteurs des forfaits regroupant le transport, l'hébergement et les visites. Les forfaits «week-end» en montagne, par exemple, pour le ski ou pour le spa, ou encore combinant des thématiques de voyage à l'hébergement (carnavals ou routes thématiques, notamment), constituent aussi des tout inclus. Bref, c'est le produit ultime pour les marchés de masse (Atherton, 1994 : 193).

La formule du tout inclus répond à plusieurs types de besoins :

- faciliter l'organisation logistique du voyage;

- encadrer les composantes du séjour à destination (repas, loisirs, visites);

- contrôler le choc du dépaysement (besoin d'accompagnement pour gérer la découverte de l'espace et de la culture hôte);

- et restreindre les dépenses.

Tous ces besoins, on le voit, sont liés à une forme ou une autre de sécurité (Lo et Lam, 2004 : 163; Wong et Kwong, 2004 : 582; Burke et Resnik, 1991). Cette sécurité est d'abord dans l'organisation logistique du voyage. Elle permet d'éviter les tracas de l'organisation puisqu'elle est prise en charge par des professionnels du voyage. Cette option est particulièrement bonne pour les voyageurs peut expérimentés, ne maîtrisant pas de langue étrangère ou ayant des réticences pour la nourriture étrangère (Lauring, 2013 : 218).

Parce qu'il entraîne le visiteur dans des situations inhabituelles, le voyage, du moins certains de ses aspects, force le voyageur à réagir à des valeurs qui peuvent être de nature conflictuelle avec celle de sa culture d'origine. Ce contact entre différents systèmes de valeurs peut générer divers types de réponses chez le touriste : acceptation et acquisition partielles ou complètes des valeurs et coutumes de l'autre, ou rejet. Dans ce dernier cas, il peut s'en suivre un choc (culturel). Le tout inclus sert alors de zone «libre», dépourvue de risque. Le fait de vivre dans sa bulle culturelle, à destination, permettra une meilleure gestion du choc culturel que pourraient autrement engendrer ce dépaysement et l'immersion en milieu étranger. À ce titre, le personnel attitré aux vacanciers à la destination joue un rôle clé comme médiateur entre les visiteurs et le milieu hôte. De même, les autres clients, issus du même milieu, jouent aussi un rôle semblable, quoique indirectement. Le groupe procure ainsi un sentiment de sécurité et de familiarité et sert à réduire le risque perçu (Wong et Lau, 2001) (voir illustration 3). Ainsi, la recherche de sécurité couvre l'intégrité physique et psychologique du séjour, dont le voyagiste garantit qu'elle ne sera pas compromise en milieu étranger.

Enfin, l'organisation du voyage procure de la sécurité sur le plan financier : toutes les dépenses associées au séjour étant planifiées et payées avant le départ, on évite ainsi les surprises à destination. Comparativement à d'autres modes de voyage, à la carte, notamment, le tout inclus permet aussi des économies d'échelle, absorbés par les entrepreneurs grâce à des coûts d'opération moins élevés. En tout inclus, les touristes seront invités à laisser leur portefeuille dans le coffre de leur chambre et, du coup, les soucis financiers. 
ILLUSTRATION 4 : On reproche souvent aux touristes de tout inclus de ne pas s'intéresser «aux autres». Et si le tout inclus servait d'abord à se rapprocher de «l'autre»?

(photo : Alain A. Grenier).

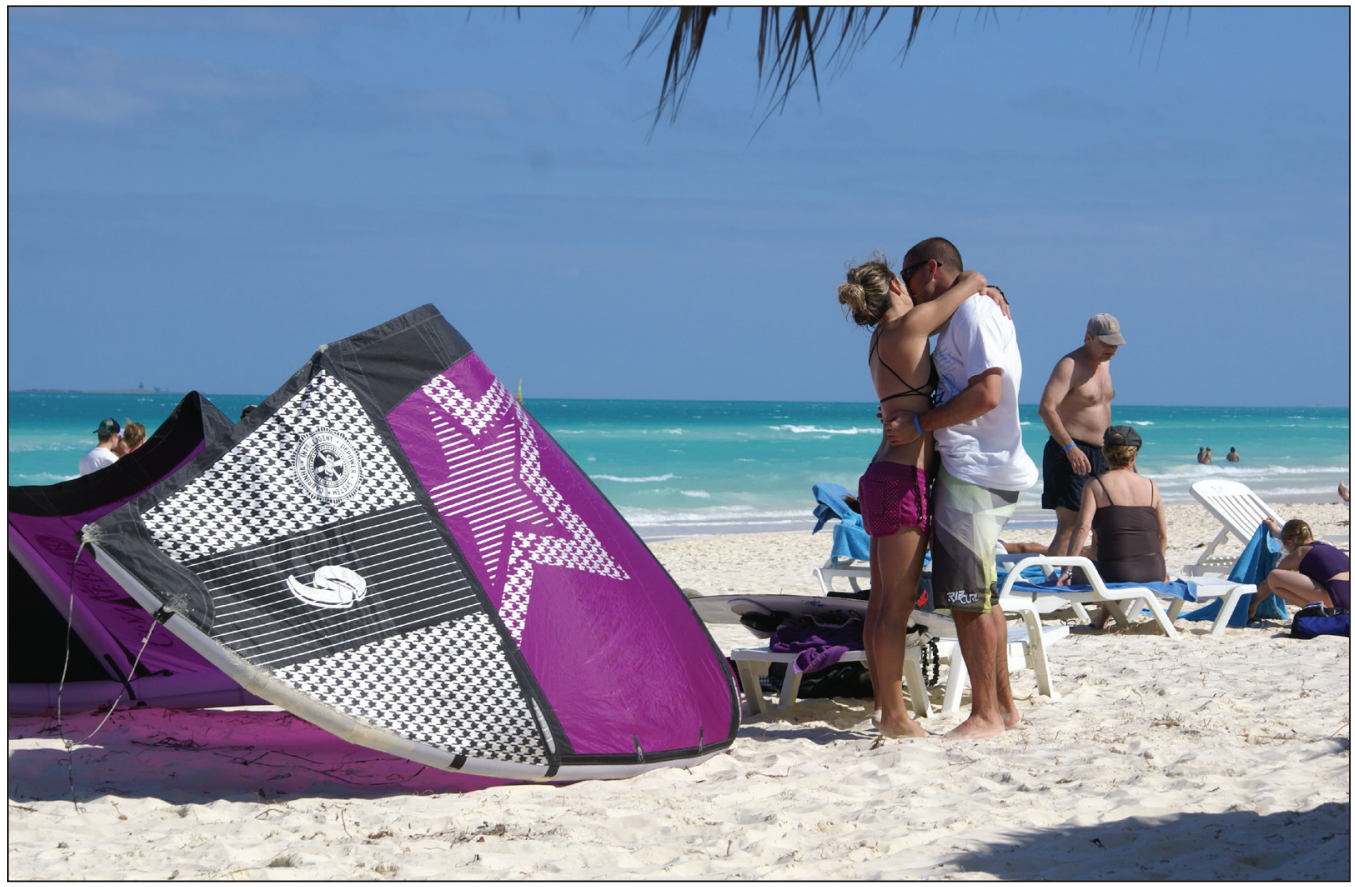

Le concept de tout inclus émerge donc en opposition au tourisme «à la carte» où le visiteur doit débourser à la pièce pour chaque service, forçant d'aucuns à être aux aguets quant à la gestion du budget. Le modèle repose donc sur une approche économique (budgétaire) du voyage, et aux besoins de sécurité socio-culturelle que procure l'enclavement en terrain étranger.

En répondant aux besoins des vacanciers en matière de sécurité, le tout inclus se révèle davantage orienté vers le consommateur que sur l'hôte - ce qui correspond à l'une des principales sources de critique de ce tourisme. Cette observation permet néanmoins d'émettre l'hypothèse que le touriste de tout inclus n'est par conséquence peut-être pas tant stimulé par la recherche de dépaysement et d'exotisme au contact d'une culture étrangère que par l'obtention d'une expérience personnelle de repos et, souvent, de rapprochement avec l'être aimé (voir illustration 4).

Traduit en infrastructure, le concept de tout inclus se présente sous trois principaux types de centres de villégiature, selon qu'ils sont ouverts, semi-ouverts ou fermés (le vase clos). Lillustration 5 juxtapose les deux grands modes de voyages (indépendant versus organisé) et détaille, pour la seconde option, les types de forfaits de voyages et d'infrastructures des tout inclus.

On peut distinguer les principaux types de forfaits de voyage selon qu'ils sont entièrement planifiés par le voyagiste (le tout inclus - transport, hébergement, repas) ou semistructurés (le forfait à la carte). Le tout inclus est payé entièrement avant le départ tandis que le forfait à la carte nécessitera des déboursés tout au long du séjour.

Quant aux types de centres de villégiature, on peut qualifier certains sites «d'ouverts " parce qu'ils ne sont pas exclusifs à un seul voyagiste et qu'ils partagent un accès à la mer avec les résidents locaux. Le site de villégiature «semi-ouvert» dispose d'une plage exclusive, mais offre un accès aux résidents locaux à la plage et des forfaits payant pour l'accès aux infrastructures (restaurants et piscines). À l'autre bout du spectre, le tout inclus «fermé» est réservé à un seul voyagiste (et, sauf exception, à une clientèle linguistique précise). Ce tout inclus est généralement géré sous forme de communauté emmurée où l'accès est interdit aux résidents locaux.

Dans les quatre cas, on y propose une vie de vacances simple pour le visiteur : soleil, sable, confort et divertissement; nourriture, breuvages et alcool à profusion, concentrés sur un site piétonnier. Cette vie paradisiaque nécessite une grande organisation et une gestion minutieuse par l'hôte. Dans le cas du tout inclus, tout est ainsi réglé au quart de tour : prise en charge des clients dès leur arrivée à la destination, transfert en autocar vers l'hôtel, inscription et «marquage» des clients (qui devront porter un bracelet identifiant le forfait choisi), et désignation des chambres. Outre la possibilité de participer à des excursions hors site, la vie à l'intérieur du centre de villégiature s'organise autour des repas, de la relaxation sur la plage ou à la piscine, et du "spectacle» en soirée.

Par sa prise en charge du client, le tout inclus offre la tranquillité d'esprit. Celle-ci résulte de l'absence de risque financier et du sentiment de sécurité que procurent l'enclavement plus ou moins hermétique du vacancier (selon le modèle choisi). Cependant, cet enclavement a un prix : celui de la déconnexion du véritable milieu hôte, et un risque relativement élevé de vivre son séjour dans sa propre bulle culturelle. Ainsi, les vacanciers qui voulaient oublier la maison y sont rappelés constamment par la présence de compatriotes qui partagent les mêmes références culturelles. La répulsion des uns constituera la sécurité des autres. Le tout inclus n'est pas à une contradiction près. 


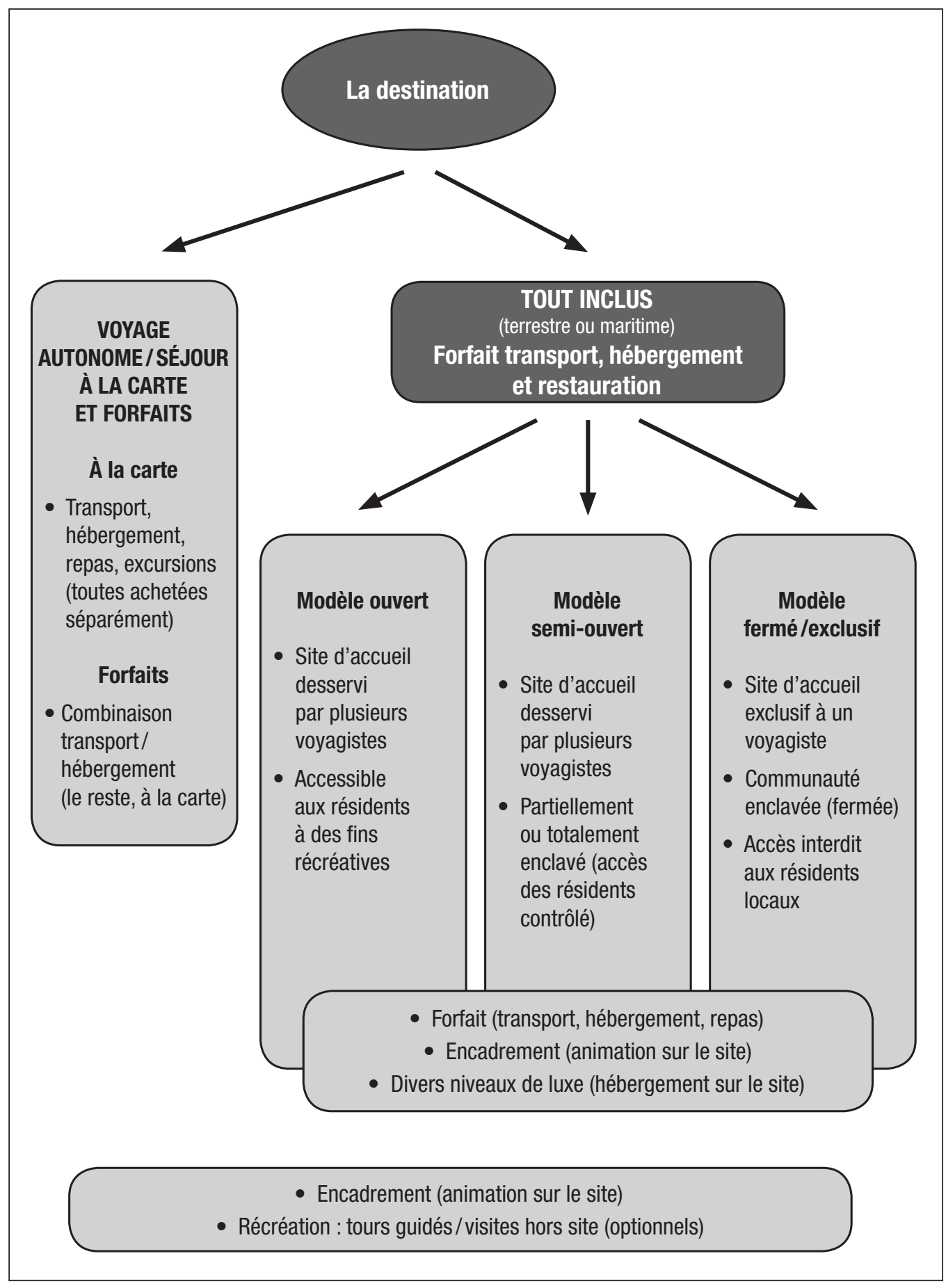

ILLUSTRATION 5 : Les formes de voyages organisés et les modèles de tout inclus (source : compilation de l'auteur).

Malgré le déclin de popularité du tout inclus observé par certains chercheurs (Larsen, 2008; Yamamoto et Gill, 1999 : 135; Urry, 1995; CTC, 1995), notamment en faveur d'expériences plus individualisées comme le tourisme de nature (souvent encore confondu dans le sillon de l'écotourisme), le tout inclus demeure bon an, mal an le produit le plus en vogue pour les vacanciers davantage intéressés par le repos que les tracas que nécessitent la planification logistique d'un voyage individuel.
Il fait l'affaire du couple - le marché dominant du tout inclus. Sa formule s'adapte aux plus aux jeunes, à l'occasion des relâches scolaires, au printemps (et souvent de pair avec la basse saison hôtelière). Le marché des célibataires n'est pas en reste : taux de divorce et autres conséquences du vieillissement de la population obligent. La diversification des groupes sociaux se reflète aussi dans l'offre, avec des complexes hôteliers tout inclus qui se spécialisent pour rejoindre des 
ILLUSTRATION 6 : Le mariage sur la plage, dans un tout inclus, offre l'exotisme du paradis terrestre, et génère la curiosité des autres vacanciers (photo : Alain A. Grenier).

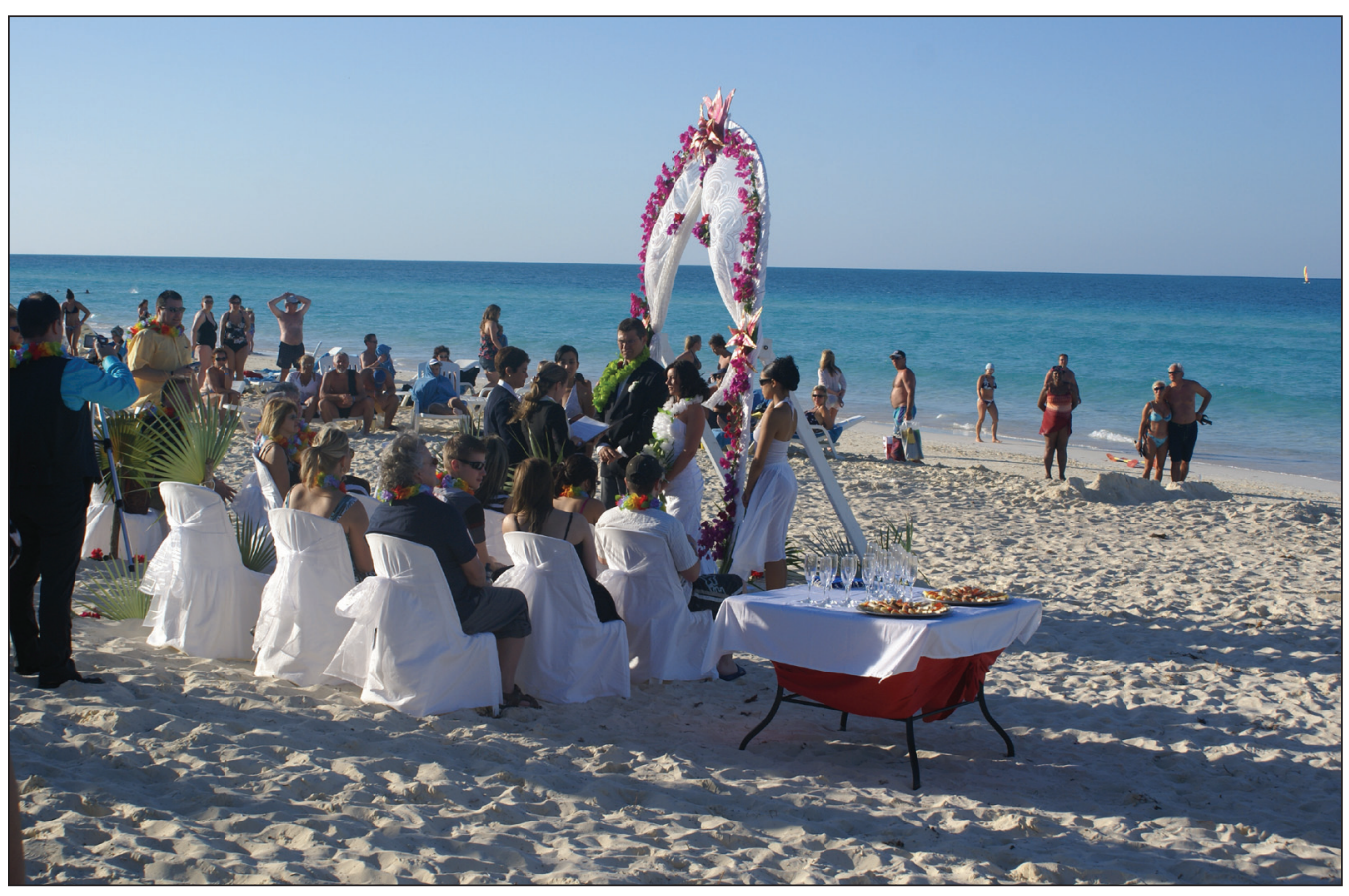

clientèles particulières : couples avec enfants, sans enfants, adultes seulement (pour favoriser les rencontres ou assurer la tranquillité, selon la lecture, ou parfois pour offrir un milieu de vacances socialement sécuritaire (tourisme des gais, lesbiens, transsexuels et transgenres)). On innove aussi par des services axés sur le mode de vie de ces divers marchés, en association avec des clubs de golf, par exemple, ou pour des rituels comme les cérémonies de mariage ou de renouvellement de vœux sur la plage (voir illustration 6).

Le tout inclus se renouvelle même en complémentarité des tourismes à la mode, comme le tourisme de nature où il délaisse son milieu balnéaire d'origine pour s'implanter en forêt pluviale, le Costa Rica, par exemple, ou dans les climats plus nordiques comme en Laponie finlandaise. Avec sa grande capacité d'adaptation, le tout inclus maintient ainsi sa fonction de produit le plus accessible pour la majorité des voyageurs, toutes classes socio-économiques confondues. Ainsi, avec plus d'un milliard de touristes de par le monde (OMT, 2013), il serait futile de croire que les tourismes alternatifs pourront à eux seuls contrer les effets négatifs du tourisme de masse. L'écotourisme et le tourisme communautaire, pour ne nommer que ceux-là, ne sont viables qu'à petite échelle - et encore. N'est-il pas ainsi plus efficace de participer à l'amélioration de la formule de masse qu'à sa simple critique? Malgré une compétitivité féroce et des clients toujours plus exigeants (Issa et Jayawardena, 2003), le tout inclus n'est pas appelé à disparaître pour la simple et bonne raison qu'il représente toujours le produit ultime pour les masses de vacanciers (Atherton, 1994 : 193). Toutefois, en réponse à ses détracteurs, le modèle du tout inclus (et des autres formes de voyages organisés) doit être revu, notamment en relation avec la durabilité sociale, culturelle et environnementale de la formule, de ses services et infrastructures. Ce dossier spécial de Téoros souhaite contribuer à baliser la voie.

\section{Dans ce dossier}

Avec ce dossier, Téoros innove une fois encore - le tourisme n'avait encore jamais été abordé dans nos pages sous l'angle du tout inclus - avec un dossier qui, s'il critique le modèle, nous rappelle que le tout inclus n'est pas le résultat d'un complot contre les populations d'accueil : il est le reflet des sociétés occidentales modernes, auxquelles nous appartenons.

Nous ouvrons le dossier avec une discussion au cœur du sujet avec deux textes qui remettent en question la légitimité du tout inclus. Le premier article, "Le "tout inclus" : contrôle du risque et de la durabilité» de Laura GIOBBI, de l'université Roma Tree, d'Italie, positionne le tout inclus dans ce tourisme post-moderne, fruit de la société de consommation, qui, segmenté et flexible, offre la possibilité aux individus «de construire leurs propres biographies [...] en fonction des souhaits individuels », et ce, «sans les contraintes de la société traditionnelle»(Giobbi, dans cette publication). Dans ses propres mots, Giobbi explique «comment, dans la période actuelle, le "tout inclus" prend une signification différente qui répond au climat d'incertitude qui entoure l'individu postmoderne au niveau individuel et collectif. Pleinement conscient de son statut ludique, en payant le voyagiste, le touriste du tout inclus obtient un séjour contrôlé et planifié qui lui permet de vivre une expérience sans risque mais qui rend difficile, en retour, une véritable connaissance mutuelle entre les peuples».

Et voilà que se pose la question qui anime toutes les discussions sur le tourisme de masse dans sa version la plus programmée : le tout inclus peut-il être une composante durable du développement touristique de toute destination?

Au Sénégal, les dommages causés par ce tourisme balnéaire de masse, auquel appartient le modèle du tout inclus, font réagir. Intitulé «Le tourisme sénégalais à la recherche d'une 
nouvelle identité», l'article de Mamadou DIOMBERA, chef du Département de tourisme de l'Université de Ziguinchor, se présente en deux volets. Dans un premier temps, elle nous décrit le gâchis de plusieurs décennies de développement touristique axées uniquement sur l'économie. Face à un déclin important, la destination n'a d'autre choix que de tenter de réparer les erreurs du passé. Elle le fait par le biais de la recherche d'une nouvelle identité touristique. Dans ce cas, elle choisit le tourisme de nature, saveur durable (le soi-disant «écotourisme»). Ce choix n'est pas lui-même sans controverse, une question qui dépasse largement le contexte de l'article. Cependant, au-delà de ce dernier aspect, nous retenons que le cas sénégalais est loin d'être unique. L'article aurait sans doute pu prendre le nom de plusieurs destinations soleil de par le monde qui, dans le contexte du nouveau paradigme du développement et de la gestion durable, s'interrogent sur leur avenir.

Comme argumenté dans cette présentation, on aurait tort de demander l'abolition du tout inclus. Si le concept est loin d'être parfait, il offre néanmoins une forme de contrôle des masses de vacanciers qui parcourent les plages de ce monde. Il nécessite aussi une meilleure compréhension quant à son expérience, ses représentations, son imaginaire et ses mythes.

C'est ce à quoi s'attaque Boualem KADRI, de l'École des sciences de la gestion de l'UQAM, en équipe avec Djaouida HAMDANI KADRI, de l'École des langues de l'Université du Québec à Montréal. Leur article intitulé «Discours publicitaire et mythologie touristique - Une analyse sémiologique des mythes du Club Med» s'intéresse à l'instrumentalisation de la mythologie par l'industrie du tout inclus. Kadri et Hamdani se penchent plus spécifiquement sur la construction de la mythologie du tourisme de tout inclus. À partir de l'analyse d'un corpus de brochures publicitaires, les auteurs illustrent comment, en cette époque de désenchantement, le tourisme "participe d'une façon soutenue à un réenchantement du monde par un imaginaire mythologique» (Kadri et Hamdani, dans cette publication) et surtout «comment cette organisation, pour compenser le réductionnisme mythologique, construit à travers son discours un système montrant une relation étroite entre le touriste-consommateur, la marque Club Med et la destination touristique».

Comme l'auront souligné les précédents articles, le tout inclus se nourrit de la perception paradisiaque des voyageurs quant aux stations balnéaires des différents continents. De fait, elles se trouvent toutes, sans exception, de part et d'autre de l'équateur, là où les courants d'eau chaude caressent les côtes de sable fin, érodées par le travail du temps. Pourtant, le tout inclus ne leur est pas exclusif. Comme dans la parabole de la paille et de la poutre, les critiques du tout inclus des destinations soleil oublient (voire ignorent) souvent que le modèle appartient aussi à la réalité des pas développés. Du Québec à l'Australie, le tout inclus se conjugue à d'autres géographies.

C'est ainsi que Gwendal SIMON, de l'Université Paris Est Marne-La-Vallée, nous propose de suivre un groupe de touristes finlandais et leur expérience du tout inclus... à Paris! Eh oui! Qui eut cru que la capitale du bon goût se prêtait aussi au tout inclus? À travers son article, «Le rôle des informations touristiques dans la médiatisation du territoire de visite : Le cas d'un voyage semi-organisé à Paris ", Simon questionne l'authenticité de la représentation de la destination à travers la mise en scène nécessitée pour les besoins des voyageurs. En prenant l'exemple de la diffusion des informations opérées par le voyagiste et de leurs réceptions par les touristes étrangers, Simon souligne «combien la médiatisation d'un territoire dans le cadre d'un voyage résulte d'une coconstruction entre les professionnels du voyage et des touristes eux-mêmes ».

\section{$* * *$}

Ce premier numéro consacré au tout inclus ne saurait prétendre couvrir tous les aspects des problématiques soulevées par le modèle du tout inclus. Au plus, les textes de ce dossier apportent certes un éclairage important sur le tout inclus, tout en soulignant le travail de recherche encore à faire.

Dans le sillon de la crise environnementale des dernières décennies, la médiatisation des espaces naturels a stimulé la croissance des tourismes alternatifs et, parallèlement, renouvelé la critique du tourisme plus conventionnel de masse. Le tout inclus, qui incarne mieux que tout autre la destination soleil, est devenu l'objet de la critique antitourisme et du mépris, tant d'une certaine classe de voyageurs que des analystes. Toutefois, devant les dérapages et abus de plus en plus nombreux rapportés des "bons tourismes alternatifs», nous voilà en droit de réexaminer le tourisme de forfait en destination soleil afin de voir comment ce tourisme réagit lui-même aux critiques et aux nouveaux paradigmes d'équité social, de respect des cultures et de l'incontournable développement durable. En fait, le tout inclus a-t-il appris de ces critiques? Si oui, comment, où en est-il et que reste-t-il à faire?

Les solutions aux impacts négatifs du tout inclus ne sont pas simples. Il s'agit ici de la gestion de la part principale du milliard de touristes qui parcourent le monde chaque année à des fins récréatives (OMT, 2013). Si l'on progresse vers la conscientisation des résidents de cette planète quant à leurs impacts, on ne peut compter seulement sur le bon vouloir des citoyens consommateurs pour renverser la vapeur. La lutte à la pauvreté nous pousse parfois à des décisions rapides, irréfléchies : la construction démesurée d'hôtels sous les cocotiers, au nom de l'emploi, comme la tentative de détourner ces touristes de plage vers les parcs, au nom de la soi-disant conservation de la nature. Malgré ses abus, le tout inclus n'est pas appelé à disparaître. Au contraire, il doit contribuer davantage à la lutte contre la pauvreté ainsi qu'à l'enrichissement des destinations et de leurs communautés.

À ce chapitre, les gestionnaires, les chercheuses et les chercheurs ont un rôle clé à jouer. Or, si l'on en croit la mièvre réponse de la communauté scientifique aux appels à textes des deux thématiques initialement proposées dans ce numéro (tourisme et pauvreté), il y a de quoi s'inquiéter : les deux thématiques ont généré très peu d'intérêt de la part de la communauté scientifique. J'observe aussi ce "désintérêt» chez les étudiants et étudiantes d'ici et d'outre-mer à qui j'enseigne et qui choisissent toujours, pour leurs recherches, de se pencher sur les tourismes alternatifs. Il est tout à fait regrettable que la recherche ne se nourrisse en ce moment quasi exclusivement que de toutes ces «alternatives»: 
tourisme communautaire, tourisme autochtone, etc. On s'inquiète ainsi de l'intrusion chez l'habitant — cet autochtone dont on a finalement envie que pour son folklore; on enquête sur la conquête des espaces et des espèces les plus rares et inaccessibles, dont les trophées que nous en ramenons sous formes de photographies et de recherches, parfois prétentieuses, s'apparentent d'avantages aux souvenirs de ces touristes à qui nous reprochons tous les maux.

La communauté scientifique, y compris nos étudiants et étudiantes, ne devrait-elle pas envisager la possibilité qu'il soit irréaliste de croire que la majorité des millions de touristes de ce monde opteront pour des produits alternatifs, hors des infrastructures construites pour les masses? Si tous les vacanciers cherchaient à avoir un degré relativement exclusif de contact avec les résidents des localités hôtes, leurs traditions ou leurs environnements naturels, les impacts de ce tourisme seraient multipliés. Ne faudrait-il pas considérer le tout inclus comme un modèle d'encadrement à développer et à parfaire? Nous qui s'intéressons au tourisme, ne devons-nous pas garder les yeux ouverts sur toutes les expériences touristiques : pas juste celles qui rompent avec le système établi, mais bien aussi sur celles qui font vivre l'industrie?

Consacrer quatre articles seulement à l'expérience touristique que choisissent pourtant plusieurs centaines de millions de touristes de par le monde a de quoi nous inquiéter. Si la réflexion était aussi facile que la critique, nous aurions davantage d'études sur le tout inclus, et ce segment de l'industrie et les communautés qui tentent d'en vivre, du Québec au Sénégal en passant par Paris, en tireraient davantage de bénéfices.

Merci aux collaboratrices et collaborateurs de ce dossier des plus importants. Bonne lecture. La discussion est lancée.

\section{Références}

ATHERTON, T. (1994) «Package Holidays: Legal Aspects», Tourism Management, $\mathrm{n}^{\circ}$ 15, p. 193-199.

BURKE, J. et B. RESNIK (1991) Marketing and Selling the Travel Product, Ohio, États-Unis : South Western Publishing Co. 323 p.

CTC - Canadian Tourism Commission (1995) Pleasure Travel Markets to North America: Japan Final Reports, Document préparé par Coopers and Lybrand Consulting, Ottawa : Canadian Tourism Commission.

DANMARKS STATISTIK (2009) Statistik Arbog [Statistical yearbook], København, Denmark : Danmarks Statistik. 577 p.

ENOCH, Y. (1996) «Contents of tour packages: A cross-cultural comparison ", Annals of Tourism Research, vol. 23, n 3, p. 599-616.

HENRY, Ben (1989) «All-Inclusive Hotels and Small Businesses in Jamaica», Journal of Hospitality \& Tourism Research, $\mathrm{n}^{\circ} 13$, p. 63-65.
HEUNG, Vincent C. S. et Raymond CHU (2000) «Important Factors Affecting Hong Kong Consumers' Choices of a Travel Agency for All-Inclusive Package Tours », Journal of Travel Research, vol. 39, n 52, p. 52-59.

ISSA, J. et C. JAYAWARDENA (2003) «The all-inclusive concept in the Caribbean ", International Journal of Contemporary Hospitality Management, vol. 15, n 3, p. 167-171.

LARSEN, J. (2008) «De-Exoticizing Tourist Travel: Everyday Life and Sociality on the Move», Leisure Studies, n 27, p. 21-34.

LAURING, Jakob (2013) «Creating the Tourist Product In the Opposition Between Self-Actualiztion and Collective Consumption: The Case of Charter Tourism ", Journal of Hospitality \& Tourism Research, vol. 37, $\mathrm{n}^{\circ} 2$, p. 217-236.

LO, Ada et Terry LAM (2004) «Long-Haul and Short-Haul Outbound AllInclusive package tours », Asia Pacific Journal of Tourism Research, vol. 9, $\mathrm{n}^{\circ} 2$, p. 161-176.

MIDDLETON, V. T. C. (1991) «Whither the Package Tour?», Tourism Management, $\mathrm{n}^{\circ} 12$, p. 185-192.

OMT — Organisation mondiale du tourisme (2013) «International tourism to continue robust growth in 2013 ", Organisation mondiale du tourisme, Communiqué de presse n 13006, Madrid, 28 janvier, <http:// media.unwto.org/en/press-release/2013-01-28/international-tourismcontinue-robust-growth-2013>, consulté le 26 mars 2013.

PMB - Print Measurement Bureau (2013) Enquête printemps 2013 — trois ans, Print Measurement Bureau.

SCHERRER, P.; A. ALONSO et L. SHERIDAN (2009) «Expanding the Destination image : Wine Tourism in the Canary Islands», International Journal of Tourism Research, $\mathrm{n}^{\circ}$ 11, p. 451-463.

SELÄNNIEMI, T. (2001) «Pale Skin on Playa del Anywhere : Finnish Tourists in the Liminoid South», DANS V. L. SMITH et M. BRENT (sous la direction de), Host and Guests Revisited: Tourism Issues of the 21st Century, p. 80-92. New York : Cognizant.

SHAW, G. et M. WILLIAMS (1994) Critical Issues in Tourism : A Geographical Perspective, Blackwell, Royaume-Uni : Oxford. 280 p.

URRY, John (1995) Consuming Places, Londres : Routledge. 257 p.

WONG, S., et E. LAU (2001) «Understanding the Behavior of Hong Kong Chinese Tourists on Group Tour Packages », Journal of Travel Research, vol. 40, p.57-67.

WONG, Chak-keung Simon et Wai-Yan Yan KWONG (2004) «Outbound Tourists' Selection Criteria For Choosing All-Inclusive Package Tours », Journal of Tourism Management, vol. 25, p. 581-592.

YAMAMOTO, Daisaku et Alison M. GILL (1999) «Emerging Trends in Japanese Package Tourism », Journal of Travel Research, vol. 38, p. 134-143. 